Differential expression analysis was performed with Expression Suite software and selected miRNAs candidates were validated in the validation study by real-time PCR with LNATM microRNA qPCR assays and analyzed with $2^{-\mathrm{DCt}}$ method. KrusKal-Wallis test, Dunns post-test and linear regression were used for statistical analyses.

Results: In the discovery study we were able to measure $379(50 \%)$ of the miRNAs represented in the array. We observed than 10 miRNAs (miRNA-Let-7a, miRNA-96, miRNA-381, miRNA-451a, miRNA-518d, miRNA-425-5p, miRNA-572, miRNA-190b, miRNA-708, and miRNA-1180) were expressed at the same level in RA and AMI patients but were significantly downregulated compared with controls. These 10 miRNAs were selected as potentially miRNAs associated with the increase risk of CVD in RA patients. Four of those miRNAs were expressed at very low level and were discarded for the validation study. In the validation study with 214 plasma samples from RA patients, we observed that two of the six candidate miRNAs (miRNA-425-5p and miRNA-451a) were significantly associated with cIMT. Thus, adjusted multivariable linear regression analysis showed that miRNA-425-5p and miRNA-451a independently explained $1.4 \%$ of the cIMT variability. Furthermore, adjusted regression estimates of the effect of miRNA-425-5p and miRNA-451a on cIMT were $\beta=$ $0.029 \mathrm{~mm} ; p=0.037$ and $\beta=-0.035 \mathrm{~mm} ; p=0.039$, respectively. No other miRNA candidate exhibited association with cIMT values. Furthermore we observed that miRNA-425-5p was significantly correlated with ESR ( $r=-0.136 ; p=0.024)$ and miRNA-451a with DAS28 $(r=0.19 ; p=0.003)$, ESR $(r=0.23 ; p=0.0001), \quad C R P(r=0.15 ; p=0.016)$ and fibrinogen $(r=0.28$; $p=0.0001)$. miRNAs concentrations were not affected by any of the AR treatments. No association was observed between the presence of carotid plaque and the expression level of the microRNAs tested.

Conclusion: In the present study, we have identified miRNA-425-5p and miRNA-451 as potentially miRNAs involved in the CVD risk observed in RA patients.

Acknowledgement: The authors thank the patients and healthy controls for their participation in the study

Disclosure of Interests: SILVIA PAREDES Speakers bureau: Bristol, Roche, Amgen, Pfiser, Abbvie, lilly, UCB, Delia Taverner Speakers bureau: amgen, pfiser, Bristol, Lilly, Roche, Raimon Ferre: None declared, Josep Maria Alegret Speakers bureau: Daichii, Lluis Masana Consultant for: amgen, daichii, sanofi, Speakers bureau: AMGEN, SANOFI, MYLAN, Joan Carles Vallve: None declared

DOI: 10.1136/annrheumdis-2019-eular.4331

\section{FRI0008 ADDRESSING THE DIAGNOSTIC GAP IN RHEUMATOID ARTHRITIS BY COMPLEMENTING THE SEROLOGY WITH GENETIC INFORMATION}

Isabel Gehring ${ }^{1}$, Leonid Padyukov ${ }^{2}$, Yutao Fu ${ }^{3}$, Sascha Swiniarski ${ }^{1}$,

Maryam Poorafshar ${ }^{4}$, Günter Steiner ${ }^{5}$, Lars Klareskog ${ }^{2}{ }^{1}$ Thermo Fisher Scientific, Freiburg, Germany; ${ }^{2}$ Karolinska Institute, Department of Medicine, Stockholm, Sweden; ${ }^{3}$ Thermo Fisher Scientific, Carlsbad, United States of America; ${ }^{4}$ Thermo Fisher Scientific, Uppsala, Sweden; ${ }^{5}$ Division of Rheumatology, Vienna, Austria

Background: Rheumatoid arthritis (RA) is caused by an interaction of inherited and environmental factors. The genetic component was revealed in different twin studies and the shared epitope was identified as main contributor. During several genome-wide association studies (GWAS) different genes have been highlighted as relevant for developing RA but so far, none of these have been used for a diagnostic approach to address the diagnostic gap for RA.

Objectives: The aim of this study was to identify genes within pathways relevant for developing $\mathrm{RA}$ and to combine these genetic risk factors with serologic data to improve the diagnosis of RA, especially in regards to CCP negative RA patients.

Methods: The cohort consists of 804 RA patients, 159 Disease controls and 495 Healthy individuals. Serology data were obtained using the Eli$A^{T M}$ CCP IgG, EliA RF IgM and EliA RF IgA (Phadia AB, Uppsala, Sweden) tests. The genetic measurements were performed using the NextGeneration-Sequencing technology AmpliSeq ${ }^{\text {TM }}$ on the lon GeneStudio ${ }^{\mathrm{TM}}$ instruments (Thermo Fisher Scientific, Carlsbad, USA). The combined dataset was analysed by machine learning focusing on multivariate supervised models and discrete models.

Results: The Disease Research Area database (Thermo Fisher Scientific, Carlsbad, USA) uses a proprietary algorithm to create connections between genes and a specific disease highlighting relevant genes and pathways using the available datasets from NCBI, DisGeNet, ClinVar and further databases. Based on this information we were able to identify genes and variants within relevant pathways for RA and designed a sequencing panel specific for diagnosis of RA. The results from the targeted sequencing approach and the serologic data of the patients were analysed by an algorithm resulting in an improved diagnosis.

Each variant within the algorithm has a specific weighting score resulting in a combination of protective and destructive variants, which are used together with the serology to calculate a RA risk score.

In this study the combination of genetic and serology leads to an improved sensitivity of over $10 \%$ compared to CCP alone, with a comparable specificity. This increase in sensitivity results in the identification of $>20 \%$ CCP-negative RA patients. Additionally, a genetic pattern was identified distinguishing between CCP-negative and CCP-positive RA patients. Conclusion: Usage of genetic variants in combination with serologic data improves the diagnosis of RA within the measured cohort of Caucasian RA patients. Furthermore, a genetic pattern specific for CCP negative RA patients was accentuated and could be used to improve the diagnosis of CCP negative RA patients in the future. The combination of serology and genetic information for the diagnosis of RA patients could lead to an earlier diagnosis, especially for CCP negative RA patients.

\section{REFERENCES}

[1] Svendsen AJ, Kyvik KO, Houen G, Junker P, Christensen K, Christiansen L, et al. (2013) On the Origin of Rheumatoid Arthritis: The Impact of Environment and Genes-A Population Based Twin Study. PLoS ONE 8(2): e57304.

[2] Jawaheer D, Gregersen PK. Rheumatoid arthritis: the genetic components Rheum Dis Clin North Am 2002; 28: 1-15.

[3] Okada, Y. et al. Genetics of rheumatoid arthritis contributes to biology and drug discovery. Nature 506, 376-381 (2014)

Disclosure of Interests: Isabel Gehring Grant/research support from: employee of Thermo Fisher Scientific, Employee of: Thermo Fisher Scientific, Leonid Padyukov Grant/research support from: Yes, but not for the presented study., Yutao Fu Grant/research support from: employee of Thermo Fisher Scientific, Employee of: employee of Thermo Fisher Scientific, Sascha Swiniarski Grant/research support from: employee of Thermo Fisher Scientific, Employee of: employee of Thermo Fisher Scientific, Maryam Poorafshar Grant/research support from: employee of Thermo Fisher Scientific, Employee of: employee of Thermo Fisher Scientific, Günter Steiner: None declared, Lars Klareskog Grant/research support from: Yes, but not for the presented study.

DOI: 10.1136/annrheumdis-2019-eular.7392

\section{FRI0009 MOLECULAR PROFILING OF CIRCULATING B- LYMPHOCYTES REVEALS THE SUPERIOR PERFORMANCE OF METHYLOME OVER TRANSCRIPTOME DATA FOR DISCRIMINATING RHEUMATOID ARTHRITIS PATIENTS IN AN EARLY ARTHRITIS CLINIC: IMPLICATIONS FOR TRANSLATING "BIG DATA" INTO CLINICALLY USEFUL TOOLS}

Najib Naamane ${ }^{1}$, Nishanthi Thalayasingam ${ }^{1}$, Nisha Nair ${ }^{2}$, Alexander Clar $^{3}$, Amy Anderson $^{1}$, Dennis Lendrem ${ }^{1}$, Louise Reynard ${ }^{3}$, Stephen Eyre ${ }^{2}$, Anne Barton ${ }^{2}$, John Isaacs ${ }^{1}$, Arthur Pratt ${ }^{1} .{ }^{1}$ Newcastle University, Institute of Cellular Medicine, Newcastle upon Tyne, United Kingdom; ${ }^{2}$ Manchester University, Arthritis Research United Kingdom Centre for Genetics and Genomics, Centre for Musculoskeletal Research, Institute of Inflammation and Repair, Manchester, United Kingdom; ${ }^{3}$ Newcastle University, Institute of Genetic Medicine, Newcastle upon Tyne, United Kingdom

Background: Defining optimal strategies for translating "big datasets" of transcriptome and methylome data into clinically valuable tools in RA will benefit from comparisons where potential confounders - including therapeutic background, disease phase and cell substrate heterogeneity - are controlled as far as possible.

Objectives: We have obtained paired B- and CD4+ T-lymphocyte whole genome expression and methylation data from drug-naïve early arthritis clinic patients at a single centre. Focussing on B-lymphocyte data we here ask which of these datasets has most value in discriminating early RA - and the additive value of combining them.

Methods: CD19+ B-lymphocytes were isolated by positive selection from fresh peripheral blood of 90 drug-naïve patients attending the Newcastle Early Arthritis Clinic (NEAC), comprising 36 RA patients and 54 disease controls matched, so far as possible, for age, sex and acute phase response. Paired RNA and DNA extracted. Gene expression was profiled using the Human HT12 v4 BeadChip, and DNA methylation at $>850,000$ $\mathrm{CpG}$ sites quantified with the MethylationEPIC array (both Illumina). Gene expression and/or DNA methylation classifiers for RA prediction were developed based on a combined approach of classification algorithm 